\title{
Review Article \\ On the Accuracy of Asymptotic Solutions for TM Waves Diffracting on an Impedance Wedge
}

\author{
Sonia Fu, ${ }^{1}$ Pierre Jacolot, ${ }^{2}$ Tommaso Balercia, ${ }^{2}$ Christian Rom, \\ Jørgen B. Andersen, ${ }^{1}$ and Gert F. Pedersen ${ }^{1}$ \\ ${ }^{1}$ APNet, Department of Electronic Systems, Faculty of Engineering and Science, Aalborg University, 9220 Aalborg, Denmark \\ ${ }^{2}$ Intel Mobile Communications, 9220 Aalborg, Denmark \\ Correspondence should be addressed to Sonia Fu; sfu@es.aau.dk
}

Received 22 July 2014; Revised 6 October 2014; Accepted 20 October 2014; Published 9 November 2014

Academic Editor: Heng-Tung Hsu

Copyright (C) 2014 Sonia Fu et al. This is an open access article distributed under the Creative Commons Attribution License, which permits unrestricted use, distribution, and reproduction in any medium, provided the original work is properly cited.

The contribution focuses on the accuracy of two asymptotic solutions aimed at representing the electromagnetic field scattered by penetrable wedges. One is a heuristic manipulation of the solution for the perfect electrical conductor, and the other one is a more rigorous coefficient based on approximate boundary conditions. The results presented here extend those proposed by other authors by illustrating the accuracy of such solutions at the edge of validity of the uniform theory of diffraction. In particular, they show that the heuristic formulation can be freely applied in similar conditions, while the other might not always lead to accurate predictions.

\section{Introduction}

At high frequencies, the propagation of electromagnetic energy follows Fermat's principle [1]. This means that, for homogeneous media, it can be predicted using a ray-tracer and a specific set of asymptotic solutions aimed at representing how electromagnetic energy interacts with matter. The simplest interaction is specular reflection, which can be easily described using the results derived within the scope of geometrical optics [2]. In this contribution, however, the attention will be put on edge diffraction and, in particular, on the accuracy of two asymptotic solutions ascribed to the uniform theory of diffraction (UTD): the diffraction coefficients proposed, respectively, by Luebbers in [3] and by Tiberio et al. in [4].

Such solutions have already been discussed and analysed in [5], where the authors compared a set of results derived with the diffraction coefficients and a collection of corresponding predictions calculated using the method of moments (MOM) [6]. The conclusion was that the two coefficients can successfully portray edge diffractions. What should be observed here, however, is that the analysis was conducted using plane waves and scenarios for which

$$
k L \gg 1,
$$

where $k$ corresponds to the wavenumber and $L$ will be defined in the next section. In other words, the assessment stayed well within the constraints of the UTD. In this paper, the accuracy of the aforementioned coefficients will be verified for

$$
k L>1
$$

and cylindrical wavefronts, which constitute a more demanding context for the coefficients. In practical terms, similar conditions can be encountered in some rooftop to rooftop trajectories in urban environments.

In order to present such an analysis, the contribution is divided as follows. Section 2 introduces the scenarios that will be analyzed next. Section 3 focuses on the solutions derived using the MOM and used for comparison. Numerical results are finally discussed in Section 4 . Section 5 concludes the contribution. 


\section{Simulations Scenario}

The analysis is contextualized to the case in which ray-tracing is used to investigate the behavior of cellular networks in urban environments. For this reason, the attention will be here brought to the ultra high frequency (UHF) band, that is, to the range [ $300 \mathrm{MHz}, 3000 \mathrm{MHz}$. In particular, considering that the focus is on inequality (2), the frequency will be set to $300 \mathrm{MHz}$, which leads to the lowest possible value of $k$.

This said, the next aspects to notice are that, in the aforementioned environments, most of the scatterers are buildings and that any building can be conveniently represented as a right prism. If the attention is put on wavefronts impinging normally on a building, the generic scatterer can thus be described using its cross-section. Without loss of generality, this is here chosen to be a square with the sides of length equal to $20 \lambda$.

The scatterer is here also assumed to be made of an isotropic, linear, and homogeneous medium for which $\epsilon_{r}=5$, $\sigma=0.01 \mathrm{~S} / \mathrm{m}$, and $\mu_{r}=1$. The notation is such that $\epsilon_{r}$ is the relative permittivity, $\sigma$ the conductivity, and $\mu_{r}$ the relative permeability. In accordance with the observations in [7], the value of $\epsilon_{r}$ was selected to model a structure of dry concrete. The value of $\sigma$ was instead chosen to be the lowest order of magnitude for which, at the selected frequency, the internal losses become appreciable.

As for the scattering problem itself, the transmitter in o is at an angle $\phi^{\prime}$ with respect to the right side of the square, as shown in Figure 1. The diffraction point is the top right corner of the scatterer and its distance to $\mathbf{o}$ and the probe in $\mathbf{r}$ is expressed, respectively, by $l^{\prime}$ and $l$. Finally, the right side of the scatterer and the diffracted ray define the angle $\phi$.

Coming to the aim of this contribution, the attention will be focused on a first scenario for which $L \approx 1 \mathrm{~m}$ and a second one for which $L<1 \mathrm{~m}$, where

$$
L=\frac{l^{\prime} l}{l^{\prime}+l} .
$$

Needless to say, the form assumed by (3) depends on the fact that the analysis considers cylindrical wavefronts [8].

In the first scenario, $l$ is set equal to $1 \mathrm{~m}$ and $l^{\prime}$ to $12 \mathrm{~m}$. The angle $\phi$ varies in the range $\left[10^{\circ}, 260^{\circ}\right]$, with a spacing of 0.001 radians. In this range there are no surface waves [9]. The angle of incidence $\phi^{\prime}$ varies instead between $195^{\circ}$ and $260^{\circ}$, with a spacing of $5^{\circ}$. Such a range was selected starting from $] 0^{\circ}, 270^{\circ}$ [. The angles in $] 0^{\circ}, 135^{\circ}$ [ were discarded using the symmetry of the cross-section and the fact that the transmitter is here assumed to be a macrocellular base station, which is typically above rooftops. The part from $130^{\circ}$ to $180^{\circ}$ was instead removed from the analysis in order to focus on angles that define areas that are both illuminated and shadowed. The case in which $\phi^{\prime}$ is equal to $180^{\circ}$ was neglected simply to avoid grazing incidence. As for the range ] $180^{\circ}, 190^{\circ}$ ], it was discarded because, with $\phi$ belonging to $\left[10^{\circ}, 260^{\circ}\right]$, the probe would otherwise have an interval in which it is always directly illuminated. In the proximity of the roof, the angles belonging to $] 260^{\circ}, 270^{\circ}$ [ were finally removed from the analysis to avoid the contributions from surface waves.

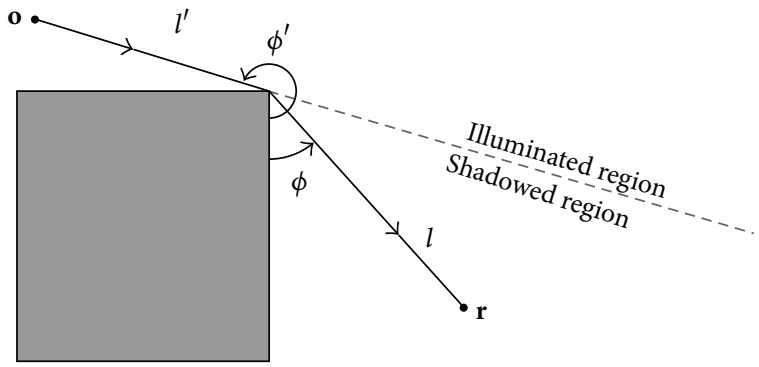

FIGURE 1: Configuration for the scattering problem.

In the second scenario, $l$ was set equal to $1 \mathrm{~m}$ and $l^{\prime}$ to $1.25 \mathrm{~m}$. The angles $\phi$ and $\phi^{\prime}$ are defined as for the previous scenario.

\section{Low-Frequency Solutions}

In order to verify the accuracy of the diffraction coefficients at hand, the total fields computed with them were here compared to the solutions calculated solving Kirchhoff's equations. All the fields refer to the case of a TM excitation. The extension to the TE case is conceptually straightforward but numerically more cumbersome and is omitted here.

3.1. EBC. Considering two media with no sources embedded in them, the exact boundary conditions (EBC) impose that the tangential components of the electric and magnetic fields remain continuous at the boundary. That is,

$$
\begin{aligned}
& \widehat{n} \times \mathbf{E}_{1}=\widehat{n} \times \mathbf{E}_{2}, \\
& \widehat{n} \times \mathbf{H}_{1}=\widehat{n} \times \mathbf{H}_{2},
\end{aligned}
$$

where the index 1 stands for the media outside the scatterer and the index 2 represents the scatterer itself.

3.2. IBC. In regards to the impedance boundary conditions (IBC), the first thing to mention is that they can be applied only when

$$
\begin{gathered}
|N| \gg 1, \\
|\mathfrak{I}(N) k a| \gg 1,
\end{gathered}
$$

where $a$ represents the smallest radius of curvature (or dimension) of the scatterer and

$$
N=\sqrt{\epsilon_{r} \mu_{r}} .
$$

When these constraints are met, the conditions impose that, in proximity of the scatterer,

$$
\widehat{n} \times(\widehat{n} \times \vec{E})=-Z_{0} \eta \widehat{n} \times \vec{H},
$$

where $\hat{n}$ stands for the outwards normal unit vector at the boundary of the scatterer, $Z_{0}$ is the free-space impedance, and

$$
\eta=\sqrt{\frac{\mu_{r}}{\epsilon_{r}} .}
$$




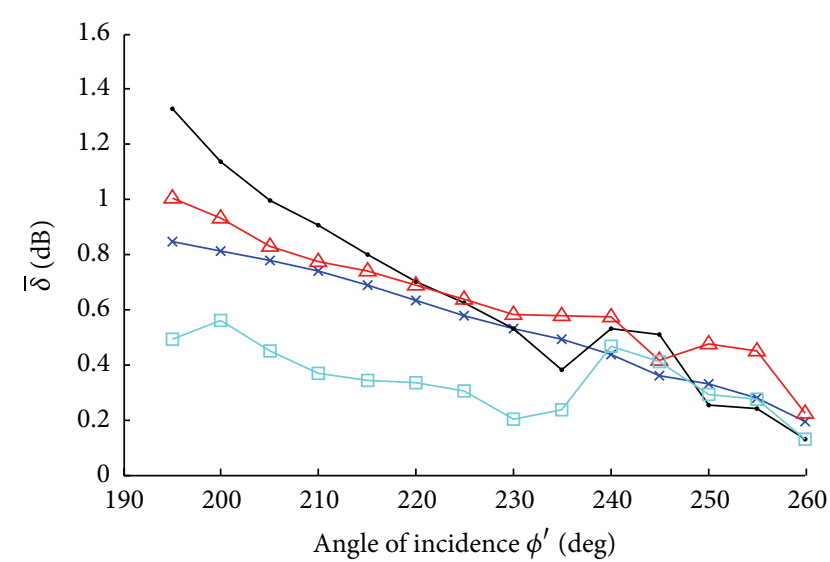

(a) Scenario 1, illuminated region

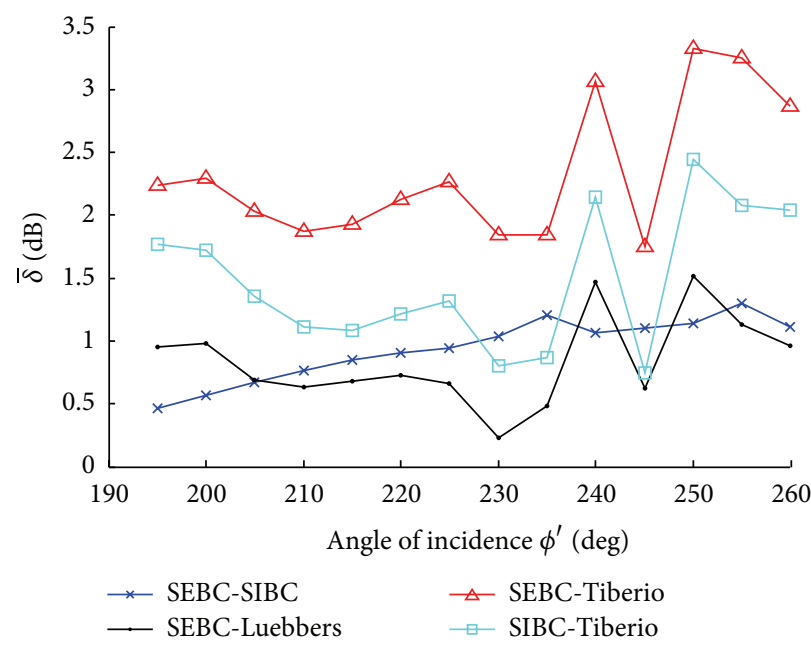

(c) Scenario 1, shadowed region

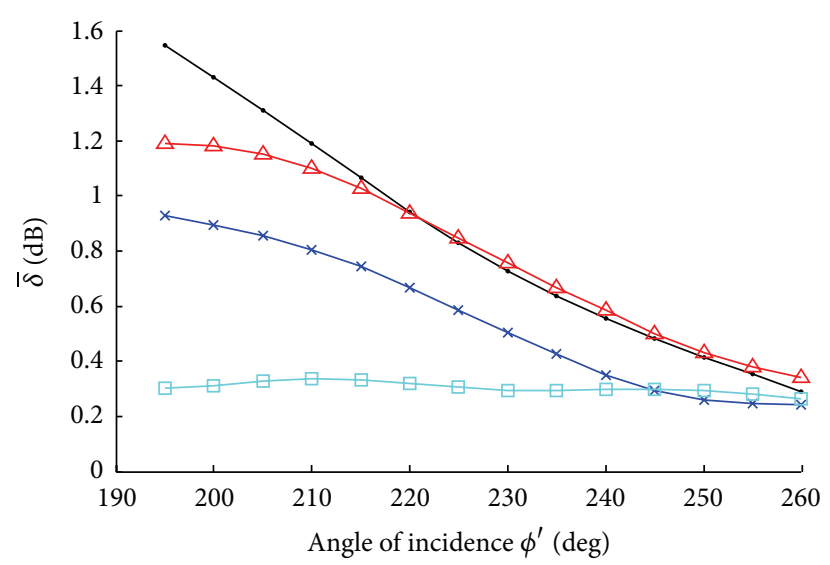

(b) Scenario 2, illuminated region

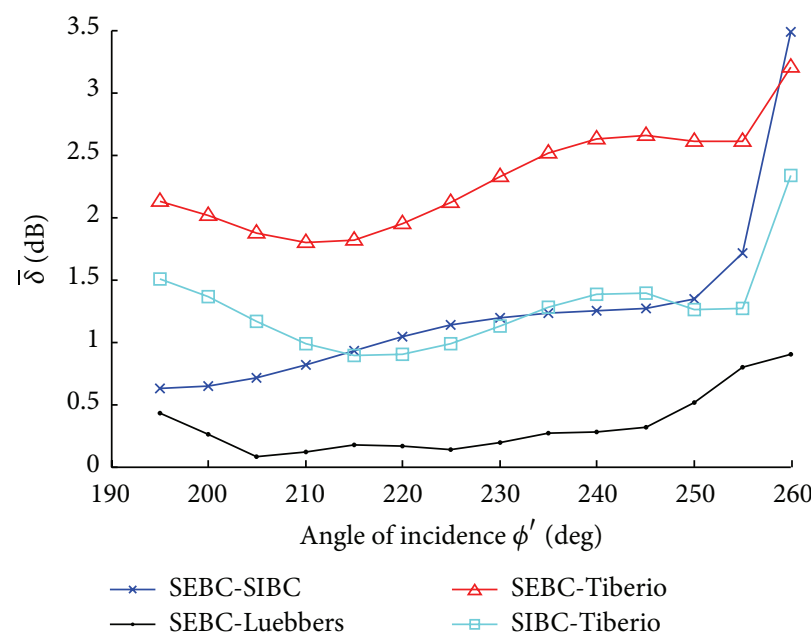

(d) Scenario 2, shadowed region

Figure 2: Trend of $\delta$ averaged over the angles of observation.

In regards to the $\mathrm{IBC}$, it is worth observing that, for the material and the geometry described in the previous section, the inequalities (6) do not apply. This contribution is thus also aimed at portraying the importance of a similar problem.

\subsection{Kirchhoff's Integral Equations Using $E B C$ and $I B C$.} Kirchhoff's integral equations are derived from Helmholtz's equation [10]. For the electric field $\vec{E}=E_{z} \cdot \vec{z}$, they can be expressed on the boundary of the scatterer, imposing

$$
\begin{aligned}
\oint_{C} & {\left[E_{z}(\bar{\rho}) \frac{\partial G\left(\bar{\rho}, \bar{\rho}^{\prime}\right)}{\partial n^{\prime}}-G\left(\bar{\rho}, \bar{\rho}^{\prime}\right) \frac{\partial E_{z}\left(\bar{\rho}^{\prime}\right)}{\partial n^{\prime}}\right] d l^{\prime}+E_{z}^{i}(\bar{\rho}) } \\
& =\frac{1}{2} E_{z}(\bar{\rho}),
\end{aligned}
$$

where $n^{\prime}$ corresponds to the unit vector normal to the scatterer in $\rho^{\prime}$ and oriented outwardly, $G$ is the two-dimensional Green's function, and $E^{i}$ is the incident field.

For the case in which the EBC are used, two equations are needed, the first being (10) applied on a point approaching the boundary from outside and the second being (11), which can be obtained inserting (4) into (10):

$$
\begin{aligned}
& -\oint_{C}\left[E_{z 1}\left(\bar{\rho}^{\prime}\right) \frac{\partial G_{2}\left(\bar{\rho}, \bar{\rho}^{\prime}\right)}{\partial n^{\prime}}-\frac{\mu_{2}}{\mu_{1}} G_{2}\left(\bar{\rho}, \bar{\rho}^{\prime}\right) \frac{\partial E_{z 1}\left(\bar{\rho}^{\prime}\right)}{\partial n^{\prime}}\right] d l^{\prime} \\
& =\frac{1}{2} E_{z 1}(\bar{\rho}) .
\end{aligned}
$$

For the IBC, introducing (8) into (10) yields instead

$$
\begin{aligned}
\oint_{C} & {\left[E_{z}\left(\bar{\rho}^{\prime}\right) \frac{\partial G\left(\bar{\rho}, \bar{\rho}^{\prime}\right)}{\partial n^{\prime}}+\frac{k}{j \eta} G\left(\bar{\rho}, \bar{\rho}^{\prime}\right) E_{z}\left(\bar{\rho}^{\prime}\right)\right] d l^{\prime}+E_{z}^{i}(\bar{\rho}) } \\
= & \frac{1}{2} E_{z}(\bar{\rho}) .
\end{aligned}
$$

The results presented in next section are derived using the MOM, respectively, on the system constituted by (10) and (11) (SEBC) and (12) (SIBC). 


\section{Results}

The goal of the simulations is to see how the coefficients derived by Luebbers and Tiberio et al. behave when only (2) holds. Tiberio's coefficient is based on the IBC and was derived in [4]. The results obtained with such a coefficient are here compared to those derived from the SEBC and the SIBC. For the coefficient proposed by Luebbers, the comparison is instead with the solution of the SEBC only. For completeness, the difference between the solutions of SEBC and SIBC is also analyzed. The results of this comparison show how realistic the IBC truly are.

For the two scenarios, two areas are considered in the comparison: the illuminated region and the shadowed one. The two regions can be seen in Figure 1. Finally, the differences between the solutions illustrated next are evaluated using the term

$$
\delta=\left.\left|10 \log _{10}\right| \frac{E_{1}}{E_{2}}\right|^{2} \mid .
$$

4.1. Illuminated Region. For the illuminated region, the incident field is of course dominant. The attention can consequently be focused on the scattered field. The trends of $\delta$, when averaged over the angles of observation, are shown in Figures 2(a) and 2(b). In this regard, the first thing to notice is that $\bar{\delta}$ is typically larger for the second scenario. This result is expected, since $L$ is larger in the first scenario. According to the same figures, it can also be observed that the solutions obtained with Tiberio's coefficient agree with those calculated by solving the SIBC. As for the accuracy of the coefficients, it is evident that choosing one or the other is irrelevant.

An interesting aspect to notice now is that, for both scenarios, the mean difference between the solutions of the SEBC and of the SIBC decreases with angle of incidence. Such a trend can be understood keeping in mind that, when $N$ is relatively small, the realism of the IBC changes with the angle of incidence [5]. Figure 3(a) further elaborates on the concept by focusing on three different variations of the second scenario.

In the first derived scenario, the scatterer has a conductivity $\sigma=1000 \mathrm{~S} / \mathrm{m}$. In the others, the scatterer is of octagonal shape (while remaining tangent to the original square along four of its sides) and has a conductivity equal, respectively, to $0.01 \mathrm{~S} / \mathrm{m}$ and $1000 \mathrm{~S} / \mathrm{m}$. As expected from inequalities (6), the IBC are more realistic when the scatterer has a high conductivity and is characterized by corners associated with a larger value of $a$.

4.2. Shadowed Region. For the shadowed region, Figures 2(c) and 2(d) illustrate that Luebbers' coefficient is more reliable than Tiberio's coefficient. It is also interesting to notice that now the results are not necessarily better for a larger value of L.

As for the difference between the solution of the SEBC and that of the SIBC, it should be noted that its average increases with the angle of incidence. In order to explain the trend, the attention can again be brought to the fact that, for the material at hand, the realism of the IBC changes

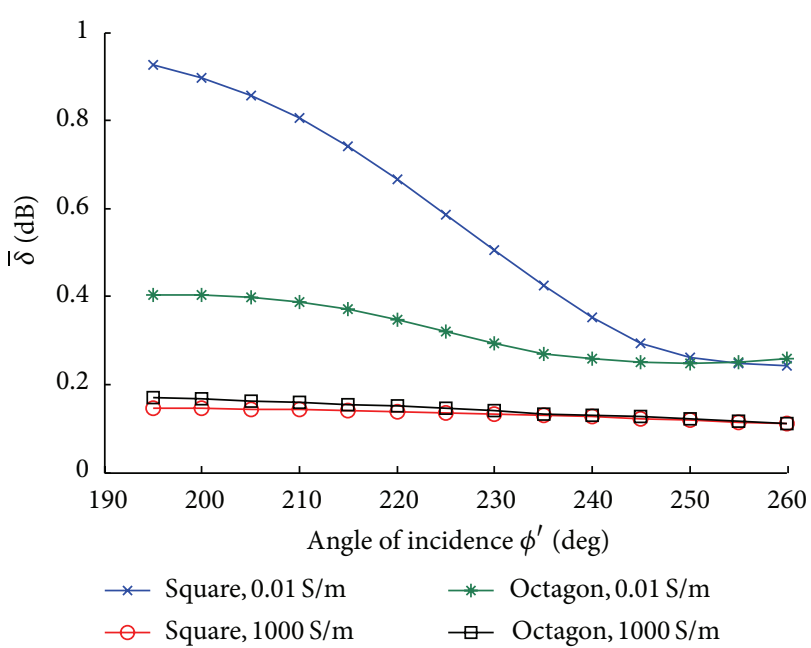

(a) Illuminated region

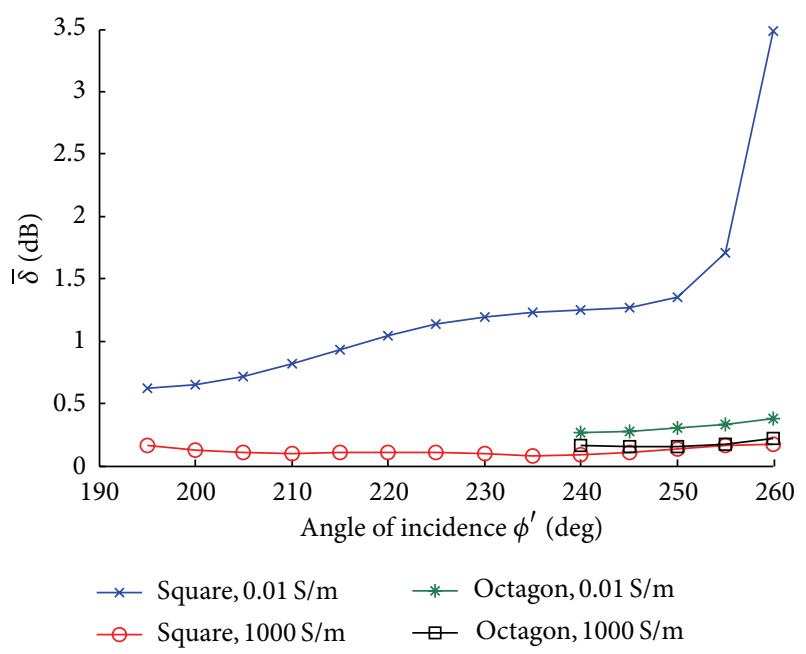

(b) Shadowed region

FIGURE 3: Average error between MOM EBC and MOM IBC for different shapes and conductivities.

with the angle of incidence. In this regard, Figure 3(b) shows that to higher conductivities and wider corners correspond again more uniform differences between the aforementioned solutions. As for how the figure was generated, it is worth mentioning that, for the octagonal cylinder, the range of observation is now $\left[55^{\circ}, 260^{\circ}\right]$. This can be attributed to the wider corners that characterize the octagon and justifies why, for the shadowed region, the curves start from $240^{\circ}$.

\section{Conclusion}

The contribution extends the work presented in [5] by analyzing the behavior of Luebbers' and Tiberio's diffraction coefficients when (1) is replaced by (2). And it does so by considering a material often encountered in simulations and focusing on the most challenging frequency in the UHF band.

With respect to the scattering problem illustrated in Figure 1, the results show that, in the illuminated region, 
the coefficients behave similarly and do not lead to major errors. For the shadowed region, instead, Luebbers' coefficient proved to return better results. In this case, it is also interesting to observe that $L$ does not have a direct impact on the quality of the results. More importantly, however, what should be noticed here is that, in such a case, inequality (6) plays a much bigger role than inequality (2) in swaying the solutions calculated with Tiberio's coefficient away from the SEBC curve. For the material analyzed here, Luebbers' coefficient can thus be regarded as a more robust asymptotic solution.

\section{Conflict of Interests}

The authors declare that there is no conflict of interests regarding the publication of this paper.

\section{References}

[1] D. A. McNamara, C. W. I. Pistotius, and J. A. G. Malherbe, Introduction to the Uniform Geometrical Theory of Diffraction, Artech House, 1990.

[2] K. Iizuka, Engineering Optics, Springer, New York, NY, USA, 3rd edition, 2008.

[3] R. J. Luebbers, "Heuristic UTD slope diffraction coefficient for rough lossy wedges," IEEE Transactions on Antennas and Propagation, vol. 37, no. 2, pp. 206-211, 1989.

[4] R. Tiberio, G. Pelosi, G. Manara, and P. H. Pathak, "Highfrequency scattering from a wedge with impedance faces illuminated by a line source. I. Diffraction," IEEE Transactions on Antennas and Propagation, vol. 37, no. 2, pp. 212-218, 1989.

[5] M. Aïdi and J. Lavergnat, "Comparison of Luebbers' and Maliuzhinets' wedge diffraction coefficients in urban channel modelling," Progress in Electromagnetics Research, vol. 33, pp. 128, 2001.

[6] H. F. Harrington, Field Computation by Moment Methods, Wiley-IEEE Press, 1993.

[7] T. Fugen, J. Maurer, T. Kayser, and W. Wiesbeck, "Capability of 3-D ray tracing for defining parameter sets for the specification of future mobile communications systems," IEEE Transactions on Antennas and Propagation, vol. 54, no. 11, pp. 3125-3137, 2006.

[8] R. G. Kouyoumjian and P. H. Pathak, "A uniform geometrical theory of diffraction for an edge in a perfectly conducting surface," Proceedings of the IEEE, vol. 62, no. 11, pp. 1448-1461, 1974.

[9] T. B. A. Senior and J. L. Volakis, Approximate Boundary Conditions in Electromagnetics, IEEE Electromagnetic Waves Series, Institution of Engineering and Technology, 1995.

[10] J. L. Volakis and K. Sertel, Integral Equation Methods in Electromagnetics, Scitech Publishing Inc, New York, NY, USA, 2011. 

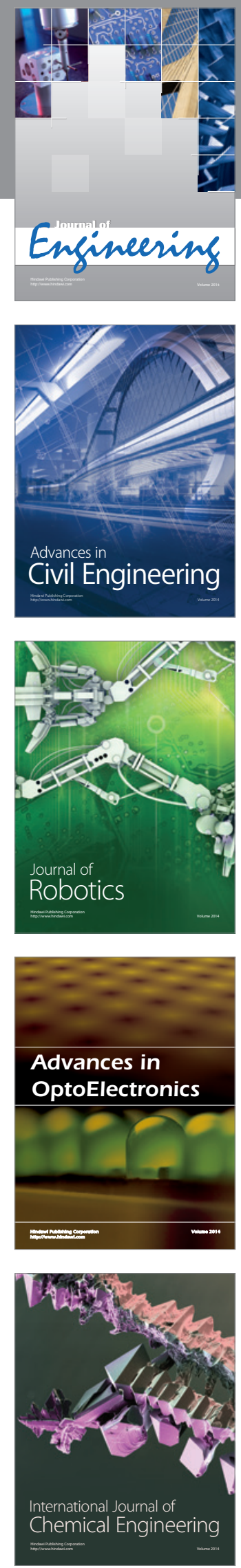

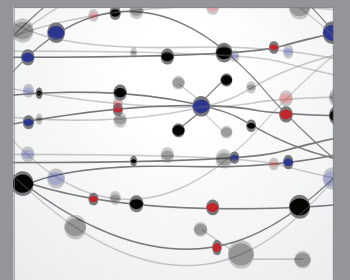

The Scientific World Journal
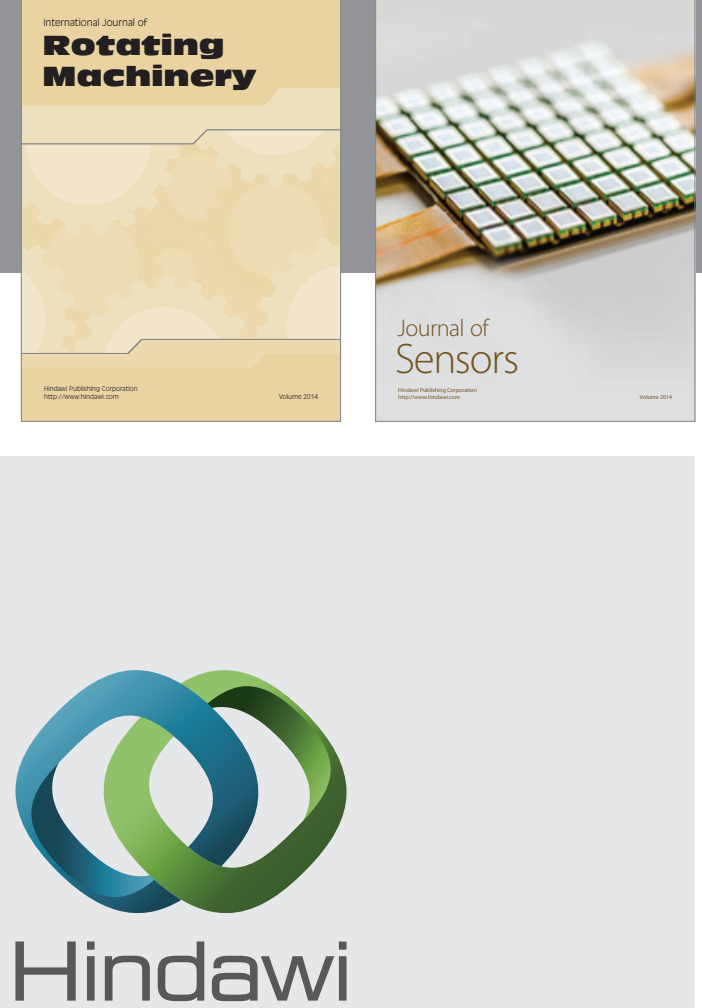

Submit your manuscripts at http://www.hindawi.com
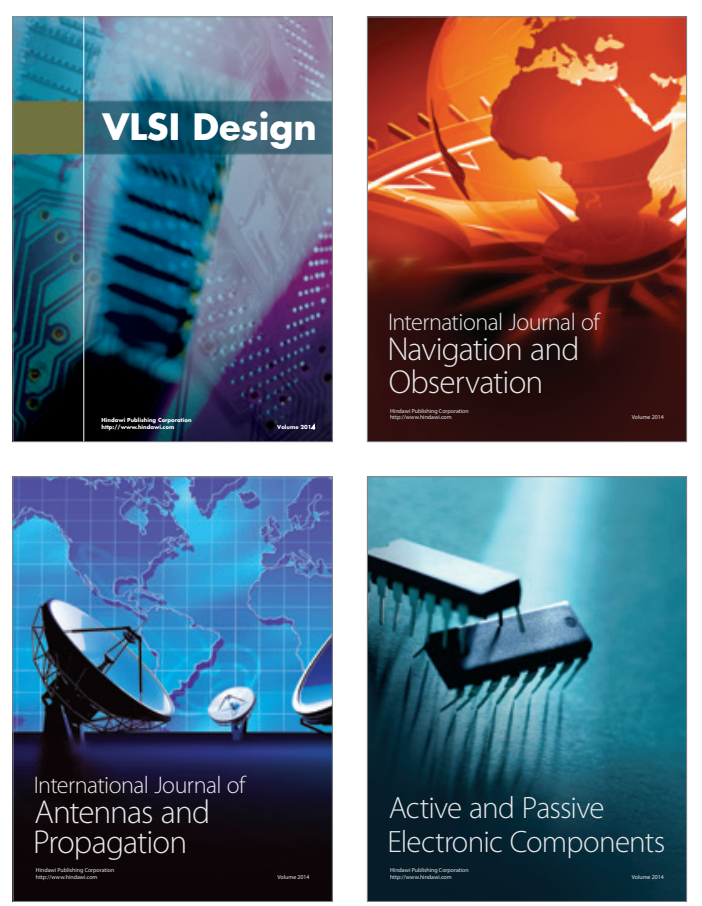
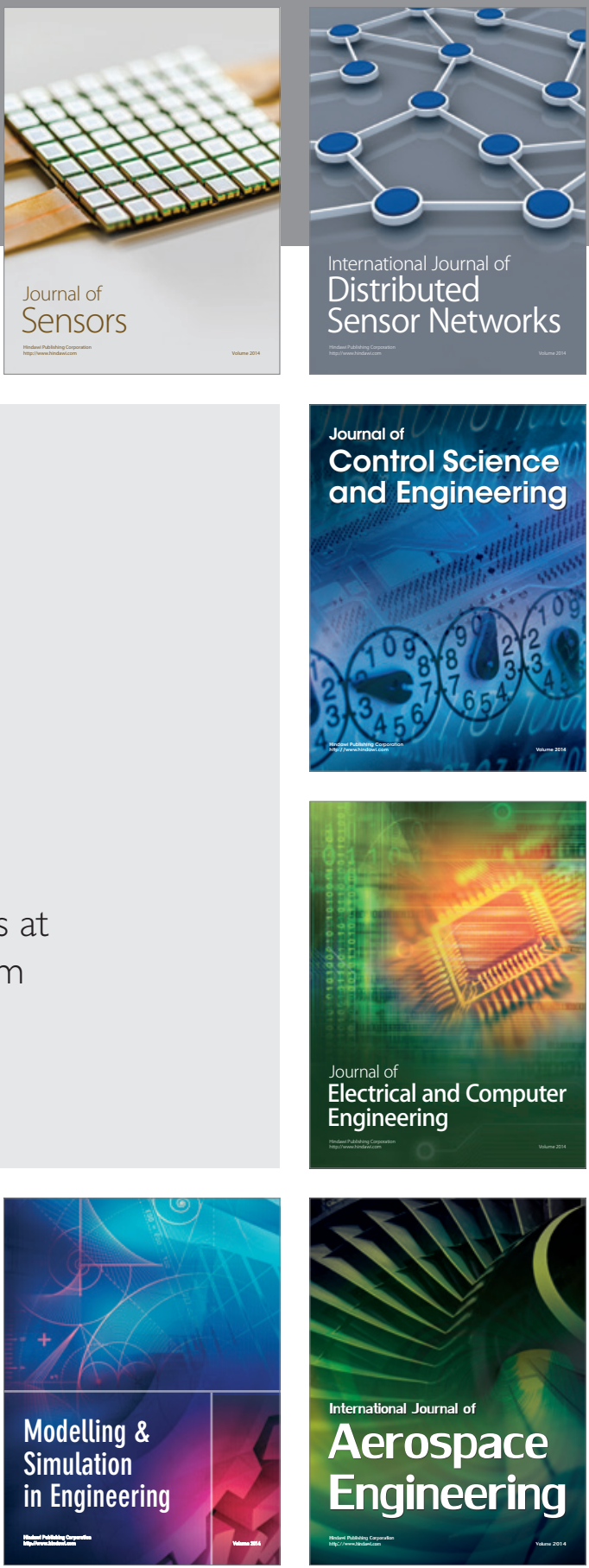

Journal of

Control Science

and Engineering
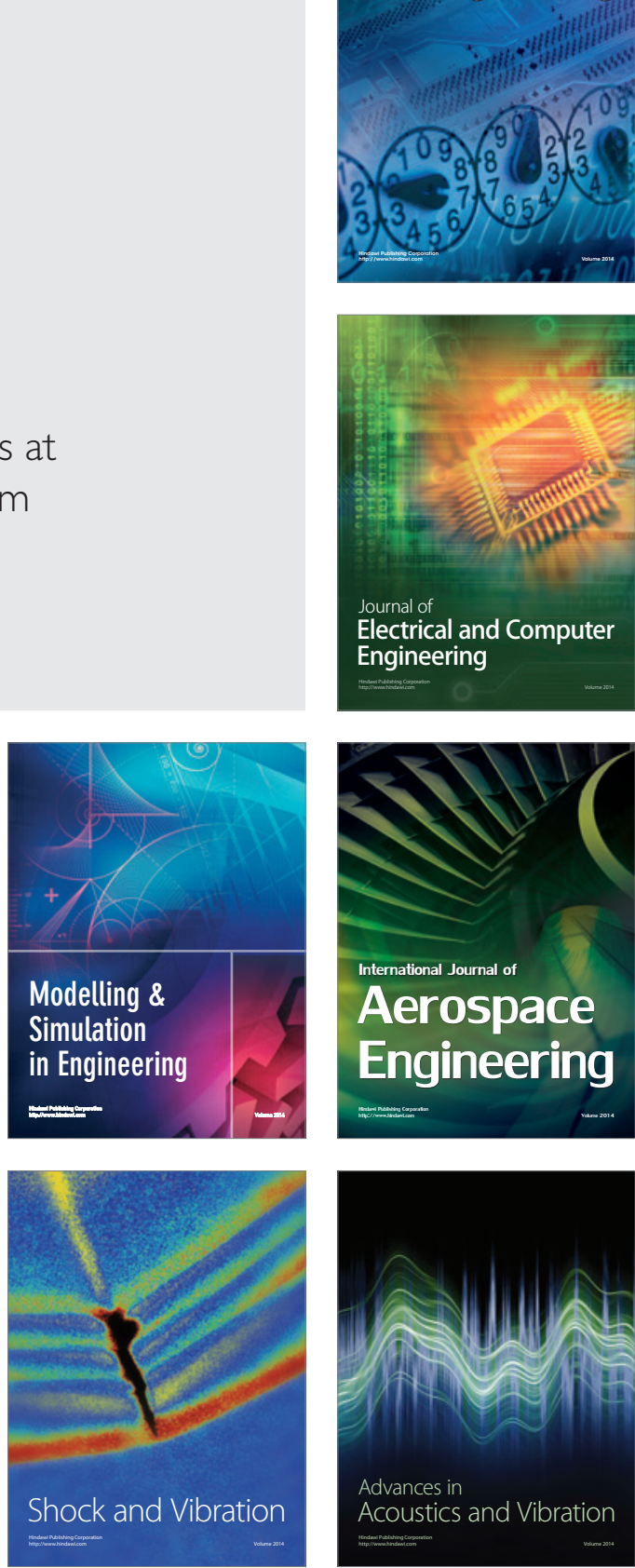